\title{
The role of iron uptake in pathogenicity and symbiosis in Photorhabdus luminescens TT01
}

\author{
Robert J Watson'1, Peter Millichap', Susan A Joyce1,2, Stuart Reynolds and David J Clarke*1,2
}

\begin{abstract}
Background: Photorhabdus are Gram negative bacteria that are pathogenic to insect larvae whilst also having a mutualistic interaction with nematodes from the family Heterorhabditis. Iron is an essential nutrient and bacteria have different mechanisms for obtaining both the ferrous $\left(\mathrm{Fe}^{2+}\right)$ and ferric $\left(\mathrm{Fe}^{3+}\right)$ forms of this metal from their environments. In this study we were interested in analyzing the role of $\mathrm{Fe}^{3+}$ and $\mathrm{Fe}^{2+}$ iron uptake systems in the ability of Photorhabdus to interact with its invertebrate hosts.
\end{abstract}

Results: We constructed targeted deletion mutants of exbD, feoABC and yfeABCD in P. luminescens TT01. The exbD mutant was predicted to be crippled in its ability to obtain $\mathrm{Fe}^{3+}$ and we show that this mutant does not grow well in iron-limited media. We also show that this mutant was avirulent to the insect but was unaffected in its symbiotic interaction with Heterorhabditis. Furthermore we show that a mutation in $f e O A B C$ (encoding a predicted $\mathrm{Fe}^{2+}$ permease) was unaffected in both virulence and symbiosis whilst the divalent cation transporter encoded by $y f e A B C D$ is required for virulence in the Tobacco Hornworm, Manduca sexta (Lepidoptera) but not in the Greater Wax Moth, Galleria mellonella (Lepidoptera). Moreover the Yfe transporter also appears to have a role during colonization of the IJ stage of the nematode.

Conclusion: In this study we show that iron uptake (via the TonB complex and the Yfe transporter) is important for the virulence of $P$. luminescens to insect larvae. Moreover this study also reveals that the Yfe transporter appears to be involved in $\mathrm{Mn}^{2+}$-uptake during growth in the gut lumen of the IJ nematode. Therefore, the Yfe transporter in $P$. luminescens TT01 is important during colonization of both the insect and nematode and, moreover, the metal ion transported by this pathway is host-dependent.

\section{Background}

Photorhabdus is a genus of Gram negative bioluminescent bacteria that are members of the Enterobacteriaceae and are therefore close relatives of important mammalian pathogens such as Escherichia coli and Salmonella. Photorhabdus have a complex life-style that involves a pathogenic interaction with insect larvae and a mutualistic interaction with nematodes from the family Heterorhabditis (for recent reviews see $[1,2]$ ). The bacteria can be normally found colonizing the gut of the infective juvenile (IJ) stage of the nematode. The IJ is a free-living, soildwelling stage of the nematode whose role is to seek out and infect susceptible insect larvae. Once inside the insect the IJ regurgitate their bacterial symbionts into the insect hemolymph and, here, the bacteria divide expo-

\footnotetext{
* Correspondence: david.clarke@ucc.ie

1 Department of Biology and Biochemistry, University of Bath, Bath BA2 7AY, UK Full list of author information is available at the end of the article
}

nentially $[3,4]$. The bacteria produce a range of activities, including hydrolytic enzymes, that contribute to the efficient conversion of the insects internal organs and tissues into bacterial biomass and the insect eventually dies of septicemia 48-72 hours post-infection [5]. At this point the IJ recovers to become an adult hermaphrodite that feeds on the bacterial biomass and lays eggs that develop through juvenile stages (L1-L4) before adulthood. After 2-3 rounds of nematode reproduction uncharacterized environmental signals stimulate the formation of an alternative L3 stage nematode called the IJ. The IJ is initially colonized by 1-2 Photorhabdus cells in a complex transmission process that has only recently been phenomonologically described [6]. These founder cells grow and divide resulting in a final population of Photorhabdus in the IJ of between 50-100 colony forming units (CFU). The IJs then emerge from the insect cadaver ready to search for more susceptible insect larvae. 
The Heterorhabditis nematode is bacteriophorous and, during growth and development, the nematode feeds on the bacterial biomass present within the cadaver. Therefore the Photorhabdus cells must be able to satisfy the nutritional requirments of the nematode population. The genetic basis of the nutritional interaction between Photorhabdus and Heterorhabditis is not well understood. There is some evidence that crystalline inclusion proteins (encoded by $\operatorname{cip} A$ and $\operatorname{cipB}$ ) produced by Photorhabdus have a role in nematode nutrition. Mutations in the $\operatorname{cip} A$ and cipB genes of Photorhabdus luminescens NC1 resulted in a strain that was unable to support nematode growth and development [7]. However the $\operatorname{cip} A$ and $\operatorname{cip} B$ mutations were pleiotropic making it difficult to confidently define a role for these proteins in nematode nutrition. Nonetheless it has been shown that overproduction of CipA and CipB in E. coli can improve the growth and development of Steinernema nematodes implying some role for these proteins in nematode nutrition [8]. A mutation in another gene, $n g r A$, encoding a phosphopantetheinyl (P'pant) trabsferase, was also shown to prevent nematode growth and development [9]. The ngrA gene was shown to be required for the production of small bioactve molecules such as siderophores and antibiotics [9]. Interestingly the stilbene antibiotic produced by all strains of Photorhabdus (3,5-dihydroxy-4-isopropylstilbene (ST)) has been shown to be important as a signal for the nematode and is involved in stimulating the recovery of the IJ to the adult hermaphrodite [10]. Moreover we have also recently shown that a mutation in the exbD gene of Photorhabdus temperata K122 was unable to support the growth and development of its nematode partner, $H$. downesi [11]. The exbD gene encodes a component of the TonB complex which is important in mediating the active uptake of siderophore-iron complexes via their cognate outer membrane receptors $[12,13]$. The defect in symbiosis of the K122 exbD mutant was rescued by the addition of $\mathrm{FeCl}_{3}$ to the media suggesting that siderophore-mediated iron uptake was important for nematode growth and development [11].

Iron is an essential nutrient that is generally found in the insoluble ferric $\left(\mathrm{Fe}^{3+}\right)$ form [14]. Many bacteria produce siderophores, molecules with very high affinities for $\mathrm{Fe}^{3+}$, in order to be able to successfully compete for $\mathrm{Fe}^{3+}$ in their environments $[15,16]$. The siderophores bind the $\mathrm{Fe}^{3+}$ and then bind to specific receptors on the surface of the bacteria. The siderophore-iron complex is then transported into the cell before the $\mathrm{Fe}^{3+}$ is reduced to $\mathrm{Fe}^{2+}$ and stored as a complex with iron-binding proteins such as bacterioferritin or used for the assembly of important cofactors such as Fe-S clusters [14,17]. Bacteria also have mechanisms to transport the low levels of ferrous $\left(\mathrm{Fe}^{2+}\right)$ iron that may be available in their environments. These transport pathways include the FeoABC permease and the YfeABCD divalent cation transporter $[14,18]$. In this study we wanted to undertake a comprehenisive analysis of the role of iron in the symbiosis between the sequenced strain of Photorhabdus (P. luminescens TT01) and its invertebrate hosts i.e. the insect and the nematode partner, $H$. bacteriophora. Therefore we constructed targeted mutants in genes predicted to play important roles in the uptake of both $\mathrm{Fe}^{3+}$ and $\mathrm{Fe}^{2+}$ and we tested these mutants for their ability to interact with the different invertebrate partners of Photorhabdus.

\section{Results}

\section{Genetic analysis of iron uptake systems in P. luminescens TT01}

We have previously shown that the exbD gene is important for both virulence and symbiosis in P. temperata (Pt) K122 [11]. The exbD gene encodes a component of the TonB complex (containing TonB, ExbD and ExbB) that is required for siderophore-mediated ferric $\left(\mathrm{Fe}^{3+}\right)$ iron uptake in many bacteria [13]. The genome sequence of $P$. luminescens (Pl) TT01 has been available since 2003 at which time it was noted that the genome contained the largest known set of iron, heme, hemin and siderophore receptors [19]. This suggested an important role for iron acquisition in the life cycle of $P$. luminescens and we decided to undertake an analysis of the role of iron uptake in the sequenced strain. In silico analysis of the genome sequence of Pl TT01 identified a single ton $B$ gene (plu2485) and a single genetic locus containing exbD (plu3940) and exbB (plu3941) (Figure 1A). To compare the role of the TonB complex in both $\mathrm{Pl}$ and $\mathrm{Pt}$ we constructed a deletion mutation in the exbD gene of Pl TT01 (the same gene that was mutated in Pt K122). It would be expected that the $\Delta e x b D$ mutant strain would be crippled for iron uptake via any siderophore-mediated pathway. In Pt K122 the exbD::Km mutation resulted in an increase in the size of the halo produced on CAS indicator agar indicating accumulation of a siderophore in the agar ([11]and Figure $1 \mathrm{~B})$. We have previously shown that this siderophore is likely to be photobactin, a catechol siderophore that was originally identified in P. luminescens NC1 $[11,20]$. Although the Pl TT01 genome is predicted to encode a variety of siderophores, it is interesting that the phb genes, encoding the proteins required for photobactin biosynthesis, are not present [19]. Moreover, the Pl TT01 $\triangle$ exbD mutation was observed to have no affect on siderophore production as observed by no change in halo size on CAS agar (Figure 1B). Therefore, Pl TT01 does not appear to be limited for iron during growth on LB agar. Nonetheless we would expect that the $\Delta$ exbD mutant would be limited in its ability to scavenge for iron under iron-limiting conditions. To test this we cultured Pl 
A)

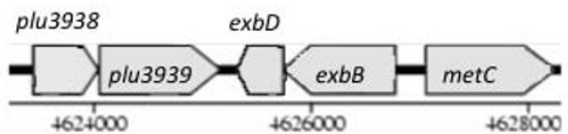

B)

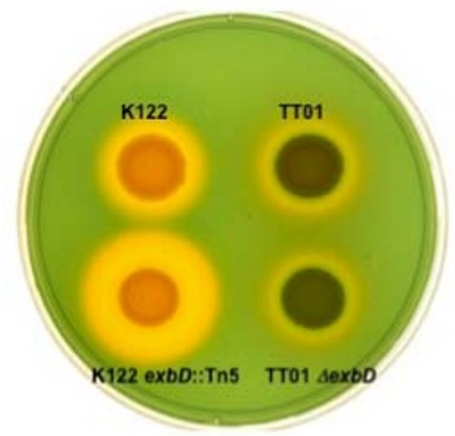

C)

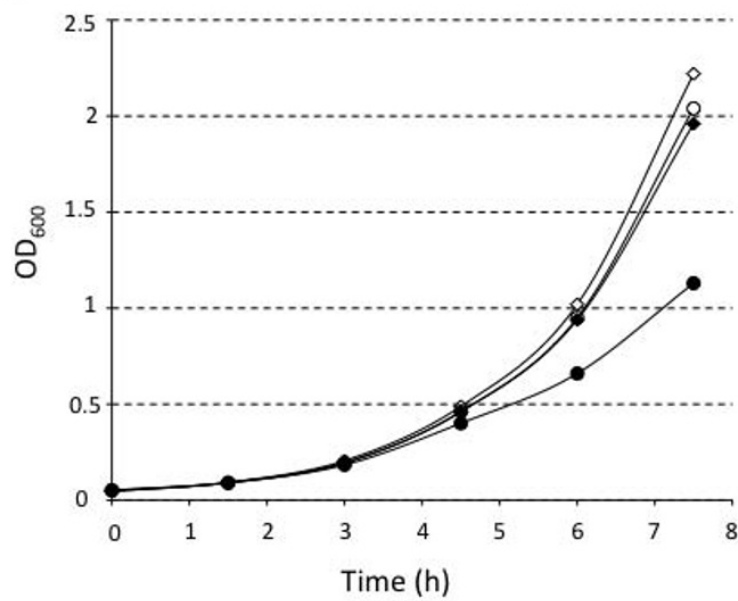

Figure 1 The exbD mutant of $P$. luminescens TT01. A) The exbD locus on the genome of $P$. luminescens $T T 01$ (taken from Colibase at http://xbase.bham.ac.uk/colibase). B) Siderophore production by $P$. temperata K122, P. temperata K122 exbD::Km, P. luminescens $T$ T01 and $P$. luminescens $T T 01 \triangle \operatorname{exbD}$. The bacteria were cultured overnight at $30^{\circ} \mathrm{C}$ in LB broth and the OD600 of the culture was adjusted to 1. An aliquot of $10 \mu$ l of each cell suspension was inoculated onto the surface of an LB agar plate containing CAS solution and the plate was incubated at $30^{\circ} \mathrm{C}$ for $48 \mathrm{~h}$. Siderophore production is observed as the orange halo surrounding the growing colony. C) The growth of $P$. luminescens $T T 01$ $\triangle e x b D$ is sensitive to the levels of iron in the medium. TT01 (diamonds) and the $\triangle$ exbD mutant (circles) were grown in fresh LB (open symbols) or LB broth supplemented with 50 MM 2'2'-dipyridyl (filled symbols). Growth curves were done in triplicate and a representative curve is shown.

TT01 and the $\triangle e x b D$ mutant in LB supplemented with 50 $\mu \mathrm{M}$ 2'-2'-dipyridyl (DIP), an iron chelator, and measured growth (Figure 1C). In the absence of DIP, the growth curves of both the WT and the $\triangle e x b D$ mutant were identical. However, in the presence of DIP, it was clear that the $\triangle \operatorname{exbD}$ mutant grew at a slower rate than the WT con- firming that the $\Delta e x b D$ mutant was less efficient at scavenging iron.

Bacteria can also utilize the small amounts of soluble ferrous $\left(\mathrm{Fe}^{2+}\right)$ iron that are present in their environments, usually in a manner that is independent of the TonB complex. We identified genes encoding two potential TonBindependent $\mathrm{Fe}^{2+}$ uptake systems, the FeoABC system and the YfeABCD system in the Pl TT01 genome (see Table 1 and Figure 2). The FeoABC system is encoded by the $f e o A B C$ operon in which FeoB is predicted to be a GTPase directly involved in $\mathrm{Fe}^{2+}$ transport [21]. On the other hand $\mathrm{Yfe} A B C D$ is an $\mathrm{ABC}$ transporter that mediates uptake of divalent cations, including $\mathrm{Fe}^{2+}[18,22]$. To test for the role of these genes in Pl TT01 we constructed $\triangle f e o A B C$ and $\triangle y f e A B C D$ mutant strains ( $\triangle f e o$ and $\triangle y f e$ respectively). We also combined mutations to produce the double mutants $\Delta f e o \Delta y f e, \Delta e x b D \Delta y f e$ and $\Delta e x b D$ $\Delta f e o$ and an $\Delta e x b D \Delta y f e \Delta f e o$ triple mutant. These iron transport mutants were then tested for their ability to grow on iron-restricted medium i.e. LB agar supplemented with increasing levels of DIP. All strains could grow equally well in the absence of DIP and, as expected, all strains carrying the $\triangle \operatorname{exbD}$ allele showed reduced growth, compared to the WT, on media containing 100 $\mu \mathrm{M}$ DIP (Figure 3). In addition, the $y f e A B C D$ locus may also play an important role in iron uptake as the $\triangle y f e$ mutant did not grow as well as WT in the presence of 150 $\mu \mathrm{M}$ DIP. Moreover the affects of the $\Delta y f e$ and $\Delta \operatorname{exbD}$ mutations appear to be additive confirming that the Yfe $\mathrm{ABC}$ transporter and the TonB complex function independently (Figure 3). On the other hand, the $\Delta f e o$ mutant was unaffected at all concentrations of DIP suggesting that this system does not play a significant role in iron scavenging under these conditions. Interestingly the $\Delta e x b D \Delta y f e \Delta f e o$ triple mutant was still able to grow on LB agar plates (even in the presence of $50 \mu \mathrm{M}$ DIP) suggesting that Pl TT01 has additional mechanisms for scavenging iron.

\section{Role of iron uptake in pathogenicity}

To determine the affect of the iron transport mutations on virulence we injected approximately $200 \mathrm{CFU}$ of each strain into 10 Galleria mellonella larvae. Pl TT01 killed the insects in around $48 \mathrm{~h}$, as did both the $\Delta y f e$ and $\Delta f e o$ mutant strains (data not shown). On the other hand no insects injected with the $\Delta e x b D$ mutant died over the 168 $\mathrm{h}$ period of the experiment (data not shown). The $\Delta \operatorname{exb} D$ mutant was also avirulent when injected into larvae of another insect model, the Tobacco Hornworm, Manduca sexta (Figure 4). Importantly, in Manduca, the virulence of the $\Delta \operatorname{exbD}$ mutant could be rescued by the pre-injection of $5 \mathrm{mM} \mathrm{FeCl}_{3}$ into the insect (Figure 4). We have shown that the injection of $5 \mathrm{mM} \mathrm{FeCl}_{3}$ was not toxic to 
Table 1: Iron transport genes in P. Iuminescens TT01 analyzed in this study.

\begin{tabular}{llll}
\hline gene & PI annotation & score & Best hit \\
\hline tonB & plu2485 & $4 \mathrm{e}-27$ & PMI1355| tonB | P. mirabilis HI4320| TonB protein \\
exbD & plu3940 & $5 \mathrm{e}-68$ & YpsIP31758_0592| exbD | Y. pseudotuberculosis IP 31758 \\
exbB & plu3941 & $1 \mathrm{e}-79$ & ECA0358| exbB | E. carotovora SCRI1043| Biopolymer transport \\
feoA & plu0209 & $8 \mathrm{e}-27$ & b3408| feoA | E. coli K12| Ferrous iron transport protein A \\
feoB & plu0208 & 0.0 & b3409| feoB | E. coli K12| Ferrous iron transport protein B \\
feoC & plu0207 & $2 \mathrm{e}-20$ & ef|ZP_04612647.1| Yersinia rohdei ATCC 43380| FeoC \\
yfeA & plu2672 & $1 \mathrm{e}-136$ & YpsIP31758_1705| yfeA | Y. pseudotuberculosis IP 31758 \\
yfeB & plu2673 & $1 \mathrm{e}-139$ & PMI1026| sitB | P. mirabilis HI4320| Iron ABC transporter \\
yfeC & plu2674 & $1 \mathrm{e}-124$ & YpsIP31758_1703| yfeC | Y. pseudotuberculosis IP 31758 \\
yfeD & plu2675 & 1e-125 & YpsIP31758_1702| yfeD | Y. pseudotuberculosis IP 31758 \\
\hline
\end{tabular}

the insect (data not shown). Remarkably, whilst the $\Delta f e o$ mutant was equally as virulent as the WT in Manduca, the $\triangle y f e$ mutant was avirulent in this insect host (Figure 4 ). This suggests that the requirement of the $y f e A B C D$ operon as a virulence factor is dependent on the insect host. Moreover virulence of the $\Delta y f e$ mutant could be rescued by the pre-injection of $\mathrm{FeCl}_{3}$ confirming that the ability to scavenge for iron is an important virulence factor in Pl TT01 (Figure 4).

\section{Role of iron uptake in symbiosis}

In this study we wanted to determine the affect of the iron homeostasis mutations in Pl TT01 on nematode growth and development. Therefore lipid agar plates were inoculated with the strains to be tested (Pl TT01, $\Delta e x b D, \Delta y f e, \Delta f e o, \Delta e x b D \Delta y f e \Delta f e o)$ and, 4 days later, the bacterial biomass was seeded with 40 surface-sterilised $H$. bacteriophora IJs. We observed that all of the Pl TT01 mutants, even the $\Delta e x b D \Delta y f e \Delta f e o$ triple mutant, were as competent as, if not better than, the WT in their ability to support the growth and development of their nematode partner as measured by the IJ yield i.e. total number of IJs collected/number of IJs inoculated (see Figure 5). This is in sharp contrast to what we had previously observed with Pt $\mathrm{K} 122$ exbD::Km where we reported that $H$. downesi nematodes failed to reproduce on the mutant bacteria [11].

Nematode development culminates in the formation of a new generation of IJs that are colonized by the bacteria on which the nematodes have been cultured. Therefore, in order to ensure the symbiotic cycle had been completed, the IJs recovered from these symbiosis assays were

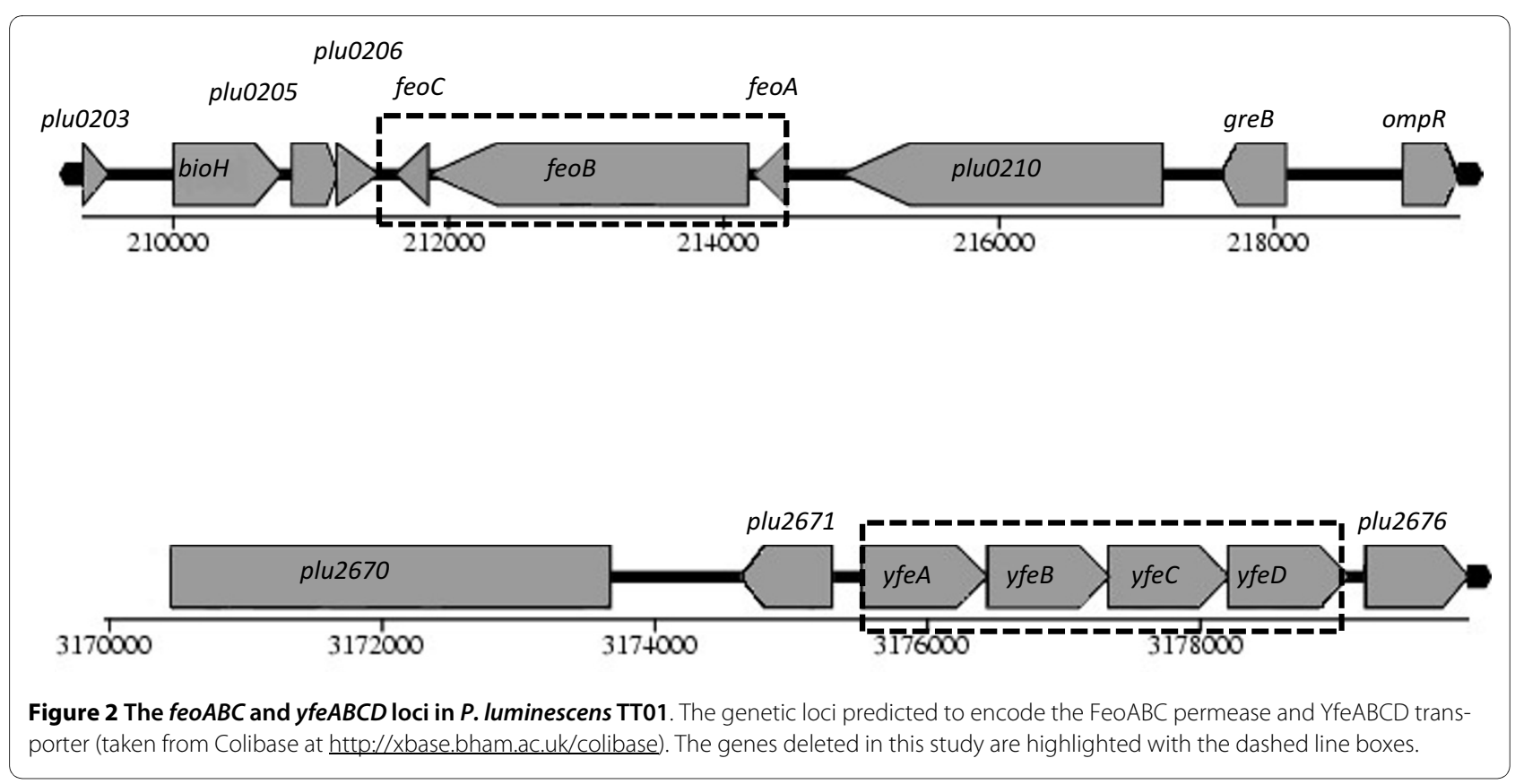




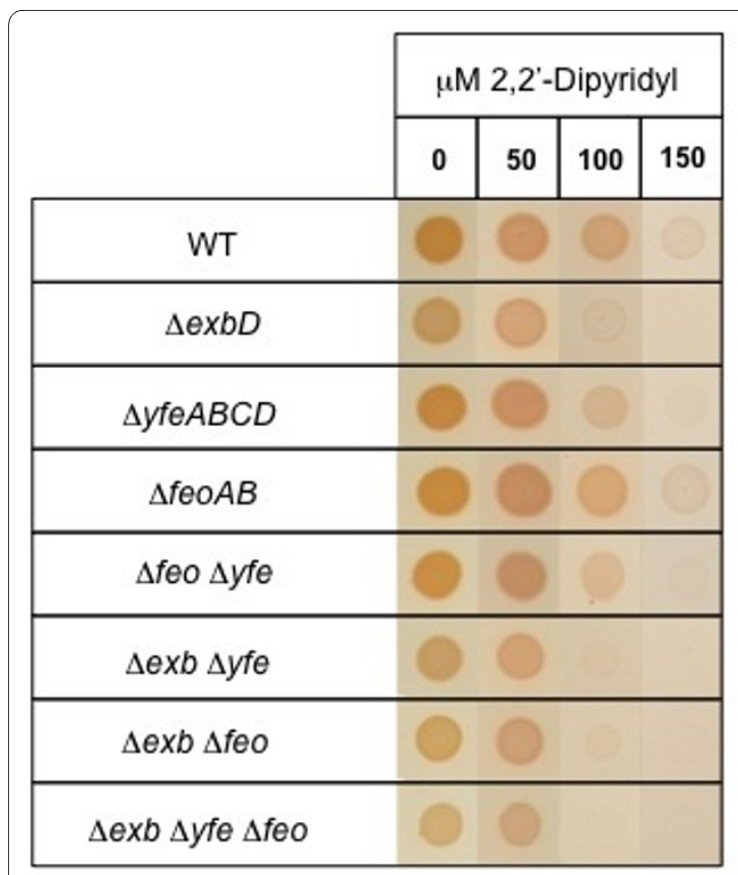

Figure 3 The growth of $P$. luminescens in the presence of 2'2'dipyridyl. The sensitivity of each mutant to iron levels was assayed by determining the ability of each mutant to grow in the presence of increasing concentrations of 2'2'-dipyridyl. The $\mathrm{OD}_{600}$ of overnight cultures of each strain was adjusted to 1 and $10 \mu$ l of the cell suspension was spotted onto the surface of an LB agar plate supplemented with the indicated concentration of 2'2'-diyridyl. The plates were incubated at $30^{\circ} \mathrm{C}$ for $48 \mathrm{~h}$ before a digital photograph of each agar plate was taken. The final image was assembled by cutting and pasting the appropriate colony from each photograph using Adobe Photoshop 7. It is important to highlight that the photographs were not manipulated in any other way. The data shown is a representative example and the experiment was repeated in triplicate.

surface sterilised, crushed individually and the lysate was spread onto LB agar. In this way it was determined that there were, on average, $42 \mathrm{CFU}$ of Pl TT01 present in the gut of each IJ (Figure 6A). Moreover the $\Delta e x b D$ and $\Delta f e o$ mutant strains were able to colonize the IJ as well as the WT (Figure 6A). However the $\Delta y f e$ and $\Delta e x b D \Delta y f e \Delta f e o$ mutants appeared to colonize the nematodes at a level that was significantly lower than WT $(\mathrm{P}<0.0001)$ suggesting that the $y f e A B C D$ locus may be important during colonization of the IJ (Figure 6A).

YfeABCD (also known as SitABCD) is an $A B C$ divalent cation transporter that has been shown to transport both $\mathrm{Fe}^{2+}$ and $\mathrm{Mn}^{2+}[18,23,24]$. In addition, both YfeABCD and $\mathrm{Mn}^{2+}$ have been implicated in resistance to reactive oxygen species (ROS) [22,25]. Photorhabdus have been reported to be very sensitive to the low levels of ROS (particularly $\mathrm{H}_{2} \mathrm{O}_{2}$ ) generated in LB agar plates after exposure of the plates to fluorescent light [26]. Therefore the low numbers of CFU obtained with the $\Delta y f e$ mutant could be explained by poor plating efficiencies due to an increased sensitivity to ROS. To test this we crushed IJs grown on either Pl TT01 or $\Delta y f e$ and plated the lysate on LB agar supplemented with $0.1 \%(\mathrm{w} / \mathrm{v})$ pyruvate (a known scavenger of $\mathrm{H}_{2} \mathrm{O}_{2}$ ). There was no difference in the number of WT Pl TT01 recovered from IJs when the lysate was plated on either LB agar or LB agar supplemented with pyruvate (Figure $6 \mathrm{~B}$ ). On the other hand, the number of CFU recovered from IJs grown on the $\Delta y f e$ mutant increased to WT levels when the lysate was plated on LB agar supplemented with pyruvate (see Figure 6B). Similar results were obtained when the LB agar plates were supplemented with catalase $\left(28 \mathrm{U} \mathrm{ml}^{-1}\right)$ or if the plates were stored in the dark before use (data not shown). Therefore the $\Delta y f e$ mutant does colonize the IJ to the same level as Pl TT01 although the $\Delta y f e$ mutant appears to be more sensitive to ROS than the WT. Interestingly we did not see any difference in the sensitivity of WT or the $\Delta y f e$ mutant to ROS when the strains were grown on LB agar and exposed to $30 \%(\mathrm{v} / \mathrm{v}) \mathrm{H}_{2} \mathrm{O}_{2}$ (data not shown). Therefore the $\Delta y f e$ mutant is not inherently more sensitive to oxidative stress and the increased sensitivity to ROS appears to be dependent on growth within the IJ, suggesting a role for the YfeABCD transporter in this environment.

\section{Bioassays using $H$. downesi reveals symbiosis defect in $\mathrm{PI}$ TT01 DexbD}

We had previously shown that the exbD gene in Pt K122 was required for the growth and development of $H$. downesi [11]. In this study we report that $H$. bacteriophora grows normally on the equivalent mutation in $\mathrm{Pl}$ TT01 (Figure 5). Therefore is the H. downesi nematode more sensitive to the exbD mutation or is the Pt K122 exbD:: Km mutant less capable of supporting nematode growth and development in general? To test this we set up a set of bioassays whereby Pl TT01 $\Delta$ exbD and Pt K122 exbD::Km were incubated separately with their cognate nematode partner or the nematode partner of the other bacterium. For 14 days after inoculation we monitored nematode growth and reproduction and observed that $H$. downesi did not grow on the Pt K122 exbD::Km mutant, as expected, but did grow normally when cultured with $\mathrm{Pt}$ K122, Pl TT01 or Pl TT01 $\Delta$ exbD (Table 2). In contrast $H$. bacteriophora grew well on all strains tested suggesting that Pt K122 exbD::Km is not generally compromised in its ability to support nematode growth and reproduction. Therefore it does appear that the $H$. downesi nematode has a more stringent requirement for iron compared to $H$. bacteriophora.

\section{Discussion}

In this study we have genetically tested the role of iron uptake in the interactions between Photorhabdus and its invertebrate hosts. We have constructed targeted dele- 


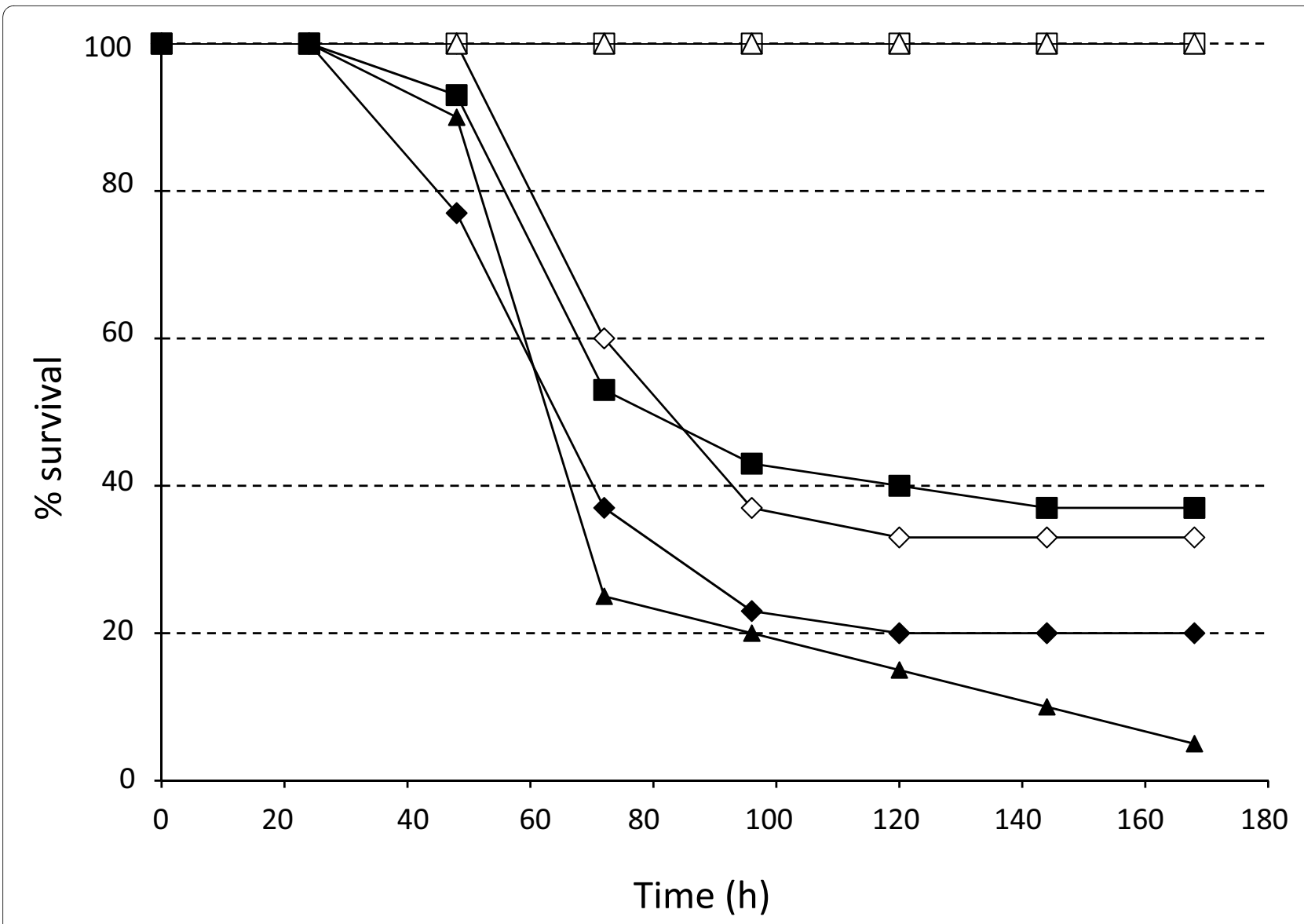

Figure 4 Virulence of the $\triangle e x b D$ and $\triangle y f e A B C D$ mutants can be rescued by $\mathrm{FeCl}_{3}$. Overnight cultures were prepared and 1000 CFU of WT (diamonds), $\triangle$ exbD (squares) and $\triangle y f e A B C D$ (triangles) were injected into $5^{\text {th }}$ instar $M$. sexta larvae (open symbols) or larvae pre-injected with $10 \mu \mathrm{l}$ of a solution containing $5 \mathrm{mM} \mathrm{FeCl}_{3}$ (filled symbols). The data shown is the mean of at least 2 independent experiments (with $\mathrm{n}=10$ insects/experiment). For clarity the standard deviations are not shown but these values were within expected limits (0-35\%).

tions of genes on the $P$. luminescens TT01 genome that are predicted to be important in both ferric $\left(\mathrm{Fe}^{3+}\right)$ and ferrous $\left(\mathrm{Fe}^{2+}\right)$ iron uptake and we have tested these mutants for phenotypes associated with virulence against insect larvae and symbiosis with $H$. bacteriophora nematodes. Our results confirm that iron uptake is important during virulence of the insect and also reveal some interesting features of the role of divalent cation uptake during the pathogenic and mutualistic interactions of Photorhabdus.

In this study we have shown that the TT01 $\Delta \operatorname{exbD}$ mutation is avirulent in the two different insect models that were tested. The exbD gene encodes for a protein that is part of the TonB complex that is found in many Gram negative bacteria. This inner membrane protein complex (composed of ExbD, ExbB and TonB) effectively transduces energy (using the proton motive force) from the inner membrane, across the periplasm, to the outer membrane [13,27]. The TonB complex interacts with outer membrane proteins (such as siderophore receptors) and the energy is used to facilitate the uptake of molecules through these outer membrane proteins. Bioinformatics can be used to identify proteins that interact with Ton $B$ based on the presence of a specific amino acid sequence called the TonB box. In this way 12 TonBdependent receptors, the majority of which $(75 \%)$ are predicted to be involved in iron uptake, have been identified in TT01 [27]. In this study we have shown that the lack of virulence associated with the $\Delta e x b D$ mutation was due to the inability of this mutant to scavenge iron within the insect environment as virulence could be rescued by the pre-injection of $\mathrm{FeCl}_{3}$. Circulating iron in the insect is bound to transferrin and it has been shown that the transcription of the transferrin gene is increased in M. sexta after a microbial challenge suggesting that reducing the availability of iron is part of the insect innate immune response (P. Millichap, unpublished data). We have previously shown that a mutation in the exbD gene of Pt K122 was attenuated in virulence due to a decrease in the growth rate in the insect [11]. Our data suggests the Pl 


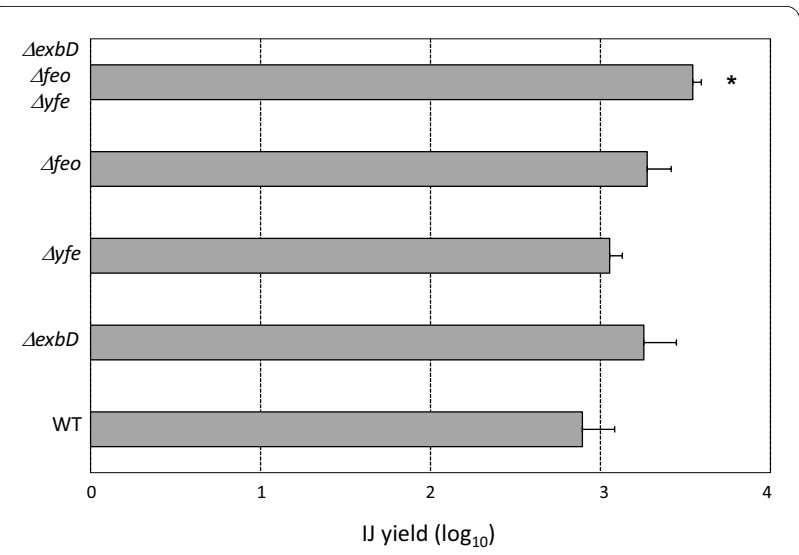

Figure 5 The IJ yield after growth on $P$. luminescens. The different bacterial strains were inoculated onto lipid agar plates, incubated for 3-4 days at $30^{\circ} \mathrm{C}$ and 40 surface-sterilised $\mathrm{H}$. bacteriophora IJs were added to the biomass. The plates were incubated for 21 days at $25^{\circ} \mathrm{C}$ and the IJ yields were determined (i.e. total number of $\mathrm{IJS} / 50$ ). For each experiment 5 plates were analyzed for each strain and the experiment was repeated 3 times. Therefore the data shown is the mean \pm standard deviation of $\mathrm{n}=15$ plates for each strain. Statistical significance was determined using a T-test and IJ yields significantly different $(\mathrm{P}<$ 0.01 ) to those obtained using TT01 are indicated with an asterisk.

TT01 $\triangle$ exbD mutant strain is unable to grow in the insect implying that Pt K122 is better at scavenging iron in the insect. Although we have not investigated the reasons for this difference we have confirmed that, similar to what has been reported in other pathogens, TonB complexmediated iron-uptake is critical for the virulence of Photorhabdus.

Nutritional interactions are one of the major driving forces in symbiotic associations [28-31] and our data suggests that iron is an important nutrient in PhotorhabdusHeterorhabditis interactions. During growth and development the nematodes feed on the bacterial biomass implying that this biomass must be able to satisfy all of the nematodes nutritional requirements, including the requirement for iron. We have previously shown that iron uptake in Pt K122 is required for the normal growth and development of $\mathrm{Hd}$ nematodes [11]. Therefore the $\mathrm{Pt}$ $\mathrm{K} 122 \operatorname{exbD::Km}$ mutant was not able to support $\mathrm{Hd}$ growth and development but this defect could be rescued by the addition of $\mathrm{Fe}^{3+}$ to the media [11]. However, in contrast to this previous work, we have now shown that the $e x b D$ gene in Pl TT01 is not required for the normal growth and development of the $\mathrm{Hb}$ nematode. Crossfeeding experiments, where the $\mathrm{Hb}$ nematode was grown on Pt K122 and the Hd nematode was grown on Pl TT01, suggested that the nematode was responsible for this difference in iron dependency as the $\mathrm{Hb}$ nematode grew equally well on the Pt K122 exbD::Km mutant and the Pl TT01 exbD mutant. In addition, although the Hd nematode was observed to grow and develop on both PI TT01 and the Pl TT01 exbD mutant, we did observe that the development of Hd IJ nematodes growing on the Pl TT01 exbD mutant was significantly delayed compared to $\mathrm{Hb}$ growing on the same bacteria (data not shown). This suggests that the Hd nematode might be more sensitive to the presence of the exbD mutation (and therefore iron levels) in their symbiotic bacteria. Such differences in sensitivity to iron levels may be one of the driving forces in the evolution and diversification of the PhotorhabdusHeterorhabditis system.

The FeoB protein is an inner membrane $\mathrm{Fe}^{2+}$ permease that requires the FeoA-dependent hydrolysis of GTP [21]. The Feo transporter is present in many bacteria and has been reported to have a role in the anaerobic-microaerophilic environment of the gastrointestinal tract of mammals. In this study we show that the FeoABC transporter has no apparent role in either the pathogenic or mutualistic life-styles of Photorhabdus. The $y f e A B C D$ operon (also found in Yersinia and annotated as sitABCD in Salmonella, Shigella and avian pathogenic Escherichia coli (APEC) and afeABCD in Actinobacillus) encodes an ATPdependent divalent cation transporter with affinity for $\mathrm{Fe}^{2+}$ and $\mathrm{Mn}^{2+}$ [32-36]. The Yfe/Sit/Afe transporter has been shown to have an important role in the virulence of many pathogens and a recent survey in $E$. coli has revealed a strong correlation between the presence of the $y f e A B C D$ operon and virulence [35]. In this study we have shown that the $y f e A B C D$ operon is important for the virulence of $P$. luminescens is some insect hosts. Therefore the $\Delta y f e$ mutant was as virulent as the WT bacteria in one lepidopteran insect host, G. mellonella, but was completely avirulent in another lepidopteran host, $M$. sexta. This implicates the $y f e A B C D$ operon as a possible hostrange determining locus in $P$. luminescens. The defect in virulence observed with the $\Delta y f e$ mutant was rescued by the pre-loading the insect with $\mathrm{Fe}^{3+}$ but not $\mathrm{Mn}^{2+}$ suggesting that the role of the Yfe transporter in insect virulence is associated with iron homeostasis (data not shown).

In this study we have also shown that the Yfe transporter may have a role during the symbiotic interaction with the nematode, in particular during the colonization of the IJ. We observed that the $\Delta y f e$ mutant has a very low plating efficiency, compared to WT, on LB agar when isolated directly from the IJ nematode. This low plating efficiency was rescued by the addition of either pyruvate or catalase, known scavengers of $\mathrm{H}_{2} \mathrm{O}_{2}$, to the LB agar plates. Therefore the $\Delta y f e$ mutant appears more sensitive to $\mathrm{H}_{2} \mathrm{O}_{2}$ than the WT bacteria. The Yfe transporter can mediate the uptake of $\mathrm{Mn}^{2+}$ and it has been shown that $\mathrm{Mn}^{2+}$ can protect the cells from ROS [18,22]. Although it was thought that part of this protective affect was due to the ability of $\mathrm{Mn}^{2+}$ to act as a chemical scavenger of ROS, recent evidence suggests that the role of $\mathrm{Mn}^{2+}$ during oxi- 
A)

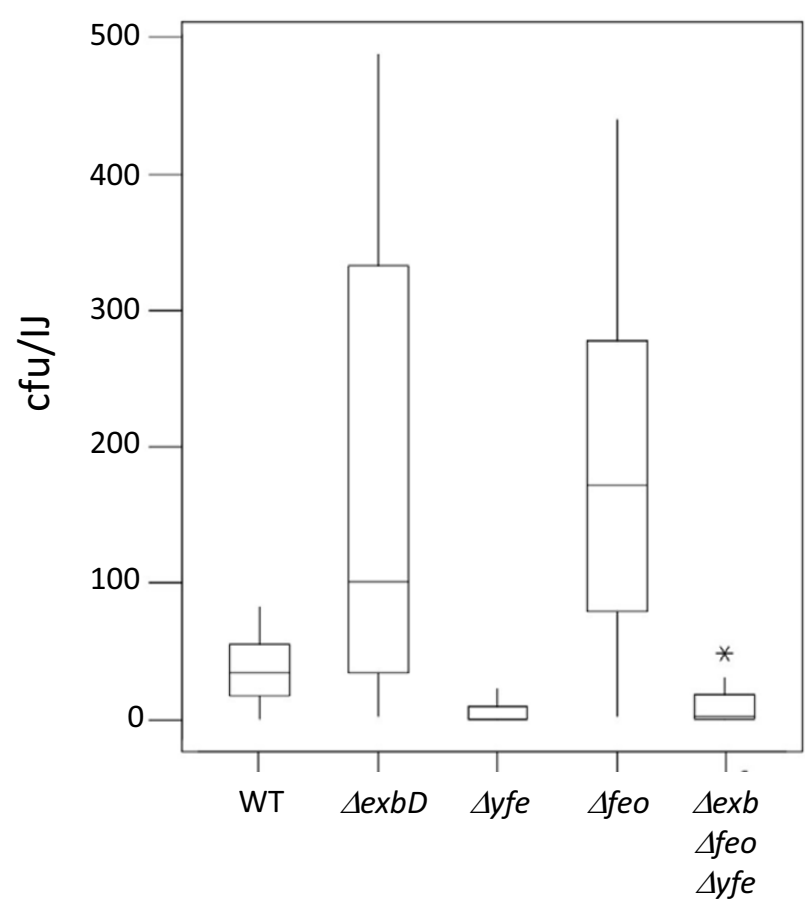

B)

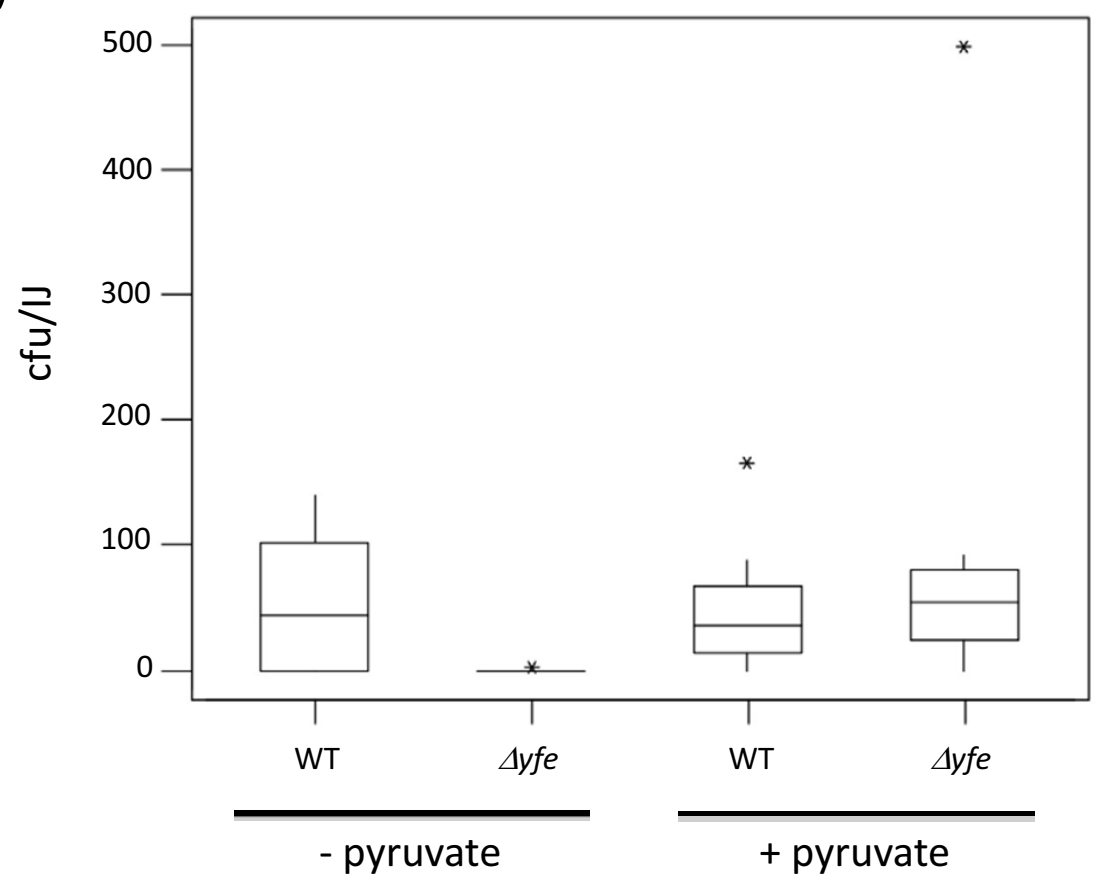

Figure 6 Colonization of IJ nematodes with TT01 and mutant derivatives. A) Individual IJ nematodes ( $n=10)$, grown on the different bacterial strains (as indicated), were crushed and the lysate was plated on LB agar to enumerate the CFU within the nematode. The data is shown as a boxplot where the horizontal line within the box represents the median value. The box extends to the first and third quartiles and the whiskers show the upper and lower limits of the data (as defined by the statistical test). Asterisks represent outliers. The level of colonization of strains carrying the $\triangle y f e A B C D$ allele was significantly lower than TT01 ( $<<0.0001$, Mann-Whitney). B) As above except that the lysate from each crushed IJ was plated on LB agar with or without added $0.1 \%(\mathrm{~W} / \mathrm{v})$ pyruvate, as indicated. 
Table 2: The growth and development of Heterorhabditis nematodes on cognate and non-cognate bacteria.

\begin{tabular}{lcc}
\hline Bacteria & Nematode growth and reproduction ${ }^{1}$ \\
\cline { 2 - 3 } & H. downesi & H. bacteriophora \\
& + & + \\
Pt K122 & - & + \\
Pt K122 exbD::Km & + & + \\
PITT01 & + & + \\
\hline
\end{tabular}

'presence (+) or absence (-) of nematode growth and reproduction after 14 days

dative stress in $E$. coli is as an enzyme co-factor (i.e. replacing the $\mathrm{Fe}^{2+}$ in $\mathrm{Fe}-\mathrm{S}$ clusters that are sensitive to oxidative stress) [25]. Many bacteria contain a dedicated Nramp-like $\mathrm{Mn}^{2+}$ transporter called MntH [18,37]. In E. coli the expression of $m n t H$ can be induced by oxidative stress and it has been reported that $m n t H$ yfe double mutants in Salmonella, APEC and Shigella are sensitive to $\mathrm{H}_{2} \mathrm{O}_{2}$ [38-40]. Therefore $\mathrm{Mn}^{2+}$ uptake appears to be critical in some cells for their ability to survive exposure to $\mathrm{H}_{2} \mathrm{O}_{2}$. Interestingly analysis of the Pl TT01 genome reveals that there is no $m n t H$ homologue in Pl TT01 and, therefore, the Yfe transporter is the only means by which Pl TT01 is predicted to be able to obtain $\mathrm{Mn}^{2+}$ from the environment. However we could not detect any inherent increase in the sensitivity of the $\Delta y f e$ mutant to $\mathrm{H}_{2} \mathrm{O}_{2}$ during growth on LB agar plates. This suggests that there is something specific about the conditions within the nematode that induces the $\mathrm{H}_{2} \mathrm{O}_{2}$-sensitive phenotype in $\mathrm{Pl}$ TT01 $\Delta y f e$. Recent studies in the model nematode Caenorhabditis elegans (a close relative of Heterorhabditis) have shown that this nematode produces 3 intestinally localized Nramp-like proteins that are involved in $\mathrm{Mn}^{2+}$ transport from the gut lumen [41,42]. Therefore, the levels of $\mathrm{Mn}^{2+}$ available to Pl TT01 within the gut of the IJ are likely to be very low. This, combined with the absence of a high-affinity $\mathrm{Mn}^{2+}$ transporter, would be expected to result in a significant reduction in the level of intracellular $\mathrm{Mn}^{2+}$ in the $\Delta y f e$ mutant that would presumably also limit the ability of the bacterium to respond to an oxidative stress.

\section{Conclusion}

In this study we show that siderophore-mediated iron uptake is important for the virulence of $P$. luminescens to insect larvae. This is similar to what has been reported for other pathogens and further highlights the relevance of Photorhabdus as a model for studying bacteria-host interactions [43]. Moreover, in contrast to what we previously reported in another species of Photorhabdus ( $P$. temperata K122) [11], we show that siderophore-mediated iron uptake in P. luminescens TT01 is not required for the growth and development of the nematode. Therefore it appears that different Photorhabdus-Heterorhabditis complexes have specific requirements for iron. In addition we show that the $y f e A B C D$ operon (encoding the Yfe divalent cation transporter) is required for virulence in some, but not all, insect hosts. Although the Yfe transporter can mediate the uptake of either $\mathrm{Fe}^{2+}$ or $\mathrm{Mn}^{2+}$ we have shown that this transporter is involved in iron uptake during pathogenicity. On the other hand we present data that suggests that the Yfe transporter may be involved in $\mathrm{Mn}^{2+}$-uptake during growth in the gut lumen of the IJ nematode. Therefore, the substrate specificity of the Yfe transporter in P. luminescens TT01 appears to be dependent on the invertebrate host colonized by the bacteria.

\section{Methods}

Bacterial strains and growth conditions

Strains used in this study are listed in Table 3. Photorhabdus temperata K122, Photorhabdus luminescens subsp laumondii TT01 and Escherichia coli strains were routinely cultured in Luria-Bertani (LB) broth or on LB agar and were incubated at $30^{\circ} \mathrm{C}$ or $37^{\circ} \mathrm{C}$ respectively. CAS agar, for the detection of siderophores, was prepared by adding CAS solution (1:10 (v:v)) into the LB agar just before pouring. CAS solution was prepared as described previously [11]. When required antibiotics were added at the following final concentrations: kanamycin $(\mathrm{Km}) 50$ $\mu \mathrm{g} / \mathrm{ml}$, ampicilin (Amp) $100 \mu \mathrm{g} / \mathrm{ml}$, chloramphenicol (Cm) $20 \mu \mathrm{g} / \mathrm{ml}$ and rifampicin (Rif) $100 \mu \mathrm{g} / \mathrm{ml}$.

\section{Construction of deletions in ex $b D$, feo $A B C$ and yfe $A B C D$}

Targeted deletion mutants were constructed as previously described [10]. Briefly paired oligonucleotides are designed to amplify approximately $500-600 \mathrm{bp}$ of DNA upstream (oligonucleotide $1+2$ ) and downstream (oligonucleotide $3+4$ ) of the gene to be deleted. The oligonucleotides used in this study are listed in Table 4. These amplicons, which have homologous terminal regions, are fused in a primerless PCR and ampliafied using oligonucleotide $1+4$ and then cloned into the suicide vector pDS132 [44]. After conjugation of the plasmid from $E$. coli S17-1 ( $\lambda$ pir) into P. luminescens TT01 exconjugants were selected by growth in the presence of $\mathrm{Cm}$ and Rif. Potential mutants were then grown overnight in LB broth and plated on LB agar with $2 \%$ sucrose to select for loss of the plasmid via a second recombination event. Sucroseresistant, chloramphenicol-sensitive colonies were then screened using colony PCR to identify mutants. Normally 
Table 3: Bacterial strains used in this study

\begin{tabular}{|c|c|c|}
\hline Strain & Genotype & Reference \\
\hline \multicolumn{3}{|l|}{ Photorhabdus } \\
\hline P. temperata (Pt) K122 & Spontaneous RifRmutant & Joyce and Clarke, 2003 \\
\hline P. luminescens (PI) TT01 & Spontaneous RifRmutant & Bennett and Clarke, 2005 \\
\hline BMM417 & $\mathrm{K} 122$ exbD::Km & Watson and Clarke, 2005 \\
\hline BMM430 & TT01 $\triangle \operatorname{exbD}$ & This study \\
\hline BMM431 ( $\Delta y f e)$ & TT01 $\triangle y f e A B C D$ & This study \\
\hline BMM432 ( $\Delta f e o)$ & ТT01 $\triangle f e o A B C$ & This study \\
\hline BMM433 & TT01 $\Delta$ exbD $\Delta y f e$ & This study \\
\hline BMM434 & TT01 $\Delta$ exbD $\Delta f e o$ & This study \\
\hline BMM435 & TT01 $\Delta f e o \Delta y f e$ & This study \\
\hline BMM436 & TT01 $\Delta$ exbD $\Delta f e o \Delta y f e$ & This study \\
\hline \multicolumn{3}{|l|}{ E.coli } \\
\hline S17-1(גpir) & lysogenised with $\lambda$ pir, replication of ori R6K & Laboratory stock \\
\hline
\end{tabular}

mutants are detected at a frequency of between 10-30\% and the amplicons from 2-3 of the colonies are sequenced to confirm the integrity of the deletion.

\section{Virulence assays}

The pathogenicity of $P$. luminescens was assessed using final instar Galleria mellonella larvae (purchased from Livefood (UK)) and freshly molted $5^{\text {th }}$ instar Manduca sexta larvae (cultured at the University of Bath) as the model insect hosts. Briefly overnight cultures of $P$. luminescens TT01 were washed 3 times in $1 \times$ PBS and the density adjusted appropriately so that $200 \mathrm{CFU}$ or 1000 CFU could be injected into the hemolymph of G. mellonella or $M$. sexta, respectively. Insects were incubated at $30^{\circ} \mathrm{C}$ and monitored for death at regular time intervals. Where appropriate insect were pre-injected with $10 \mu \mathrm{l}$ of either $5 \mathrm{mM} \mathrm{FeCl}_{3}$ or $5 \mathrm{mM} \mathrm{MnCl}_{2}$ at least $30 \mathrm{~min}$ before the bacteria were injected.

Table 4: Oligonucleotides used for construction of targeted deletion mutants.

\begin{tabular}{|c|c|c|}
\hline Gene(s) & Sequence $5^{\prime}$ to $3^{\prime *}$ & Name \\
\hline \multirow[t]{4}{*}{$e x b D$} & 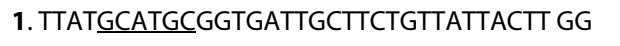 & RJW115 \\
\hline & 2. GAATCAGTGACAATTACATAAGTCACCTTGTCTTG & RJW116 \\
\hline & 3. CAAGGTGACTTATGTAATTGTCACTGATTCTTCC & RJW117 \\
\hline & 4. TTATGAGCTCGCCAACCAATITGCCTCTGCCCTAC & RJW118 \\
\hline \multirow[t]{4}{*}{$y f e A B C D$} & 1. TTATGCATGCGGTTATCAATACCTGCCAGATGC & RJW171 \\
\hline & 2. CССТTITGTTACATAAATTCAAACC & RJW172 \\
\hline & 3. GGTTTGAATTTATGTAACAAAAAGGGTTATATCTG & RJW173 \\
\hline & 4. TTATGAGCTCGGTGTTGAAGTITGTTACTTATAGC & RJW174 \\
\hline \multirow[t]{4}{*}{ feoABC } & 1. TTATGCATGCCGTAGTAAAAGCGGGTGATATCG & RJW167 \\
\hline & 2. GCTAATCATTITCAATTCCTACATATGACCTTCCG & RJW168 \\
\hline & 3. CGGAAGGTCATATGTAGGAATTGAAAATGATTAGC & RJW169 \\
\hline & 4. TTATGAGCTCCCAAAACGCTTCTCTTAGAAGATGC & RJW170 \\
\hline
\end{tabular}

${ }^{*}$ : the underlined sequence indicate the restriction endonuclease sites used for cloning the amplicon into pDS132. 


\section{Nematode growth and development}

To determine the ability of each mutant to support nematode growth and development we carried out in vitro symbiosis assays. Therefore the bacteria were cultured overnight in LB and $50 \mu \mathrm{l}$ was spread, in a $\mathrm{Z}$ pattern, onto the surface of a lipid agar plate $(/ 500 \mathrm{ml}: 12.5 \mathrm{~g}$ nutrient agar, $5 \mathrm{~g}$ corn syrup, $2,5 \mathrm{~g}$ yeast extract, $2.5 \mathrm{ml}$ cod liver oil, $1 \mathrm{~g} \mathrm{MgCl} \cdot 6 \mathrm{H}_{2} \mathrm{O}$ ) containing Rif and incubated at $30^{\circ} \mathrm{C}$ for 3-4 days. The bacterial biomass was then seeded with 40 surface-sterilised $H$. bacteriophora IJs and incubated at $25^{\circ} \mathrm{C}$ for 21 days. The presence of the Rif ensures that any bacteria present in the IJ are not able to compete with the lawn of bacteria present on the lipid agar plate. After 21 days the new generation of IJs had migrated to the lid of the Petri dish and these nematodes were collected in $1 \times$ PBS and enumerated to determine the IJ yield (i.e. total number of IJs collected/number of IJs inoculated).

\section{Colonization assay}

To determine colonization levels by each of the mutants IJs collected from the in vitro symbiosis assays were incubated at room temperature for at least 7 days before analysis. This incubation provides the bacteria with the opportunity to reproduce in the IJ gut and form a stable population. The IJs were surface-sterilised by washing in $0.4 \%(\mathrm{w} / \mathrm{v})$ hyamine and individual IJs were crushed in $100 \mu \mathrm{l}$ of PBS and the lysate was plated on LB (with or without added pyruvate). The plates were incubated at $30^{\circ} \mathrm{C}$ and the number of CFU's was determined after $48 \mathrm{~h}$.

\section{Authors' contributions}

RJW undertook all of the experiments described in this manuscript with the exception of the virulence assays in Manduca sexta (which were carried out by PM). RJW, SAJ and DJC conceived of the study. SAJ, SR and DJC designed the experiments and DJC wrote the manuscript. All authors have read and approved the final manuscript.

\section{Acknowledgements}

RJW and PM were supported by studentships from the BBSRC. SAJ was funded through the Exploiting Genomics initiative of the BBSRC (project number: 86/ EGA16183 awarded to DJC and SR). Work in the lab of DJC is currently funded by Science Foundation Ireland.

\section{Author Details}

'Department of Biology and Biochemistry, University of Bath, Bath BA2 7AY, UK and 2Department of Microbiology, University College Cork, Cork, Ireland

Received: 30 October 2009 Accepted: 22 June 2010

Published: 22 June 2010

\section{References}

1. Waterfield NR, Ciche T, Clarke D: Photorhabdus and a host of hosts. Annu Rev Microbiol 2009, 63:557-574.

2. Clarke DJ: Photorhabdus: a model for the analysis of pathogenicity and mutualism. Cell Microbiol 2008, 10:2159-2167.

3. Ciche TA, Ensign JC: For the insect pathogen Photorhabdus luminescens, which end of a nematode is out? Appl Environ Microbiol 2003, 69:1890-1897.

4. Clarke DJ, Dowds BCA: Virulence mechanisms of Photorhabdus sp strain K122 toward wax moth larvae. J Invert Pathol 1995, 66:149-155.
5. Daborn PJ, Waterfield N, Blight MA, ffrench-Constant RH: Measuring virulence factor expression by the pathogenic bacterium Photorhabdus luminescens in culture and during insect infection. J Bacteriol 2001, 183:5834-5839.

6. Ciche TA, Kim K, Kaufmann-Daszczuk B, Nguyen KCQ, Hall DH: Cell invasion and matricide during Photorhabdus luminescens transmission by Heterorhabditis bacteriophora nematodes. Appl Environ Microbiol 2008, 74:2275-2287

7. Bintrim SB, Ensign JC: Insertional inactivation of genes encoding the crystalline inclusion proteins of Photorhabdus luminescens results in mutants with pleiotropic phenotypes. J Bacteriol 1998, 180:1261-1269.

8. You J, Liang S, Cao L, Liu X, Han R: Nutritive significance of crystalline inclusion proteins of Photorhabdus luminescens in Steinernema nematodes. FEMS Microbiol Ecol 2006, 55:178-185.

9. Ciche TA, Bintrim SB, Horswill AR, Ensign JC: A phosphopantetheinyl transferase homolog is essential for Photorhabdus luminescens to support growth and reproduction of the entomopathogenic nematode Heterorhabditis bacteriophora. J Bacteriol 2001 183:3117-3126

10. Joyce SA, Brachmann AO, Glazer I, Lango L, Schwar G, Clarke DJ, Bode HB: Bacterial biosynthesis of a multipotent stilbene. Angewantde Chemie 2008, 47:1942-1945.

11. Watson RJ, Joyce SA, Spencer GV, Clarke DJ: The exbD gene of Photorhabdus temperata is required for full virulence in insects and symbiosis with the nematode Heterorhabditis. Mol Microbiol 2005, 56:763-773.

12. Braun V: Energy-coupled transport and signal transduction through the gram-negative outer membrane via TonB-ExbB-ExbD-dependent receptor proteins. FEMS Microbiol Rev 1995, 16:295-307.

13. Braun V, Braun M: Active transport of iron and siderophore antibiotics. Curr Opin Microbiol 2002, 5:194-201.

14. Andrews SC, Robinson AK, Rodriguez-Quinones F: Bacterial iron homeostasis. FEMS Microbiol Rev 2003, 27:215-237.

15. Wandersman C, Delepelaire P: Bacterial iron sources: from siderophores to hemophores. Annu Rev Microbio/ 2004, 58:611-647.

16. Faraldo-Gomez JD, Sansom MS: Acquisition of siderophores in gramnegative bacteria. Nat Rev Mol Cell Biol 2003, 4:105-116.

17. Andrews SC: Iron storage in bacteria. Adv Microb Physiol 1998, 40:281-351

18. Papp-Wallace KM, Maguire ME: Manganese transport and the role of manganese in virulence. Annu Rev Microbio/ 2006, 60:187-209.

19. Duchaud E, Rusniok C, Frangeul L, Buchrieser C, Givaudan A, Taourit S, Bocs S, Boursaux-Eude C, Chandler M, Charles JF, et al:: The genome sequence of the entomopathogenic bacterium Photorhabdus luminescens. Nat Biotechnol 2003, 21:1307-1313.

20. Ciche TA, Blackburn M, Carney JR, Ensign JC: Photobactin: a catechol siderophore produced by Photorhabdus luminescens, an entomopathogen mutually associated with Heterorhabditis bacteriophora NC1 nematodes. Appl Environ Microbiol 2003, 69:4706-4713

21. Cartron ML, Maddocks S, Gillingham P, Craven CJ, Andrews SC: Feotransport of ferrous iron into bacteria. Biometals 2006, 19:143-157.

22. Kehres DG, Maguire ME: Emerging themes in manganese transport, biochemistry and pathogenesis in bacteria. FEMS Microbiol Rev 2003, 27:263-290

23. Janakiraman A, Slauch JM: The putative iron transport system SitABCD encoded on SPI1 is required for full virulence of Salmonella typhimurium. Mol Microbiol 2000, 35:1146-1155.

24. Kehres DG, Janakiraman A, Slauch JM, Maguire ME: SitABCD is the alkaline $\mathrm{Mn}(2+)$ transporter of Salmonella enterica serovar Typhimurium. J Bacteriol 2002, 184:3159-3166.

25. Anjem A, Varghese S, Imlay JA: Manganese import is a key element of the OxyR response to hydrogen peroxide in Escherichia coli. Mol Microbiol 2009, 72:844-858.

26. Xu J, Hurlbert RE: Toxicity of irradiated media for Xenorhabdus spp. App/ Environ Microbiol 1990, 56:815-818.

27. Koebnik R: TonB-dependent trans-envelope signalling: the exception or the rule? Trends Microbio/ 2005, 13:343-347

28. Zientz E, Dandekar T, Gross R: Metabolic interdependence of obligate intracellular bacteria and their insect hosts. Microbio/ Mol Biol Rev 2004, 68:745-770 
29. Prell J, Poole P: Metabolic changes of rhizobia in legume nodules. Trends Microbiol 2006, 14:161-168.

30. Leser TD, Molbak L: Better living through microbial action: the benefits of the mammalian gastrointestinal microbiota on the host. Environ Microbiol 2009, 11:2194-2206.

31. Harrison MJ: Signaling in the arbuscular mycorrhizal symbiosis. Annu Rev Microbiol 2005, 59:19-42.

32. Rhodes ER, Menke S, Shoemaker C, Tomaras AP, McGillivary G, Actis LA: Iron acquisition in the dental pathogen Actinobacillus actinomycetemcomitans: what does it use as a source and how does it get this essential metal? Biometals 2007, 20:365-377.

33. Zhou D, Hardt WD, Galan JE: Salmonella typhimurium encodes a putative iron transport system within the centisome 63 pathogenicity island. Infect Immun 1999, 67:1974-1981.

34. Runyen-Janecky $L$, Reeves SA, Gonzales EG, Payne SM: Contribution of the Shigella flexneri Sit, luc, and Feo iron acquisition systems to iron acquisition in vitro and in cultured cells. Infect Immun 2003, 71:1919-1928.

35. Sabri $M$, Leveille $S$, Dozois $C M$ : A SitABCD homologue from an avian pathogenic Escherichia coli strain mediates transport of iron and manganese and resistance to hydrogen peroxide. Microbiology 2006, 152:745-758.

36. Perry RD, Mier I Jr, Fetherston JD: Roles of the Yfe and Feo transporters of Yersinia pestis in iron uptake and intracellular growth. Biometals 2007, 20:699-703.

37. Richer E, Courville P, Bergevin I, Cellier MF: Horizontal gene transfer of "prototype" Nramp in bacteria. J Mol Evol 2003, 57:363-376.

38. Kehres DG, Janakiraman A, Slauch JM, Maguire ME: Regulation of Salmonella enterica serovar Typhimurium $\mathrm{mntH}$ transcription by $\mathrm{H}(2) \mathrm{O}(2), \mathrm{Fe}(2+)$, and $\mathrm{Mn}(2+)$. J Bacteriol 2002, 184:3151-3158.

39. Sabri M, Caza M, ProulX J, Lymberopoulos MH, Bree A, Moulin-Schouleur $M$, Curtiss R, Dozois CM: Contribution of the SitABCD, MntH, and FeoB metal transporters to the virulence of avian pathogenic Escherichia coli O78 strain chi7122. Infect Immun 2008, 76(2):601-611.

40. Runyen-Janecky L, Dazenski E, Hawkins S, Warner L: Role and regulation of the Shigella flexneri sit and MntH systems. Infect Immun 2006 74:4666-4672.

41. Settivari R, Levora J, Nass R: The divalent metal transporter homologues SMF-1/2 mediates dopamine neuron sensitivity in Caenorhabditis elegans models of manganism and Parkinson's disease. J Biol Chem 2009, 284:35758-68

42. Bandyopadhyay J, Song HO, Park BJ, Singaravelu G, Sun JL, Ahnn J, Cho JH: Functional assessment of Nramp-like metal transporters and manganese in Caenorhabditis elegans. Biochem Biophys Res Commun 2009, 390:136-141.

43. Schaible UE, Kaufmann SH: Iron and microbial infection. Nat Rev Microbiol 2004, 2:946-953.

44. Philippe N, Alcaraz J-P, Coursange E, Geiselmann J, Schneider D: Improvement of pCVD442, a suicide plasmid for gene allele exchange in bacteria. Plasmid 2004, 51:246-255

doi: $10.1186 / 1471-2180-10-177$

Cite this article as: Watson et al., The role of iron uptake in pathogenicity and symbiosis in Photorhabdus luminescens TT01 BMC Microbiology 2010, 10:177

Submit your next manuscript to BioMed Centra and take full advantage of:

- Convenient online submission

- Thorough peer review

- No space constraints or color figure charges

- Immediate publication on acceptance

- Inclusion in PubMed, CAS, Scopus and Google Scholar

- Research which is freely available for redistribution

Submit your manuscript at www.biomedcentral.com/submit
C Biomed Central 